\title{
PART 9.
}

Outer Solar Nebula and Planetary

Atmospheres 


\title{
Chemistry in the Outer Solar System
}

\author{
Tobias C. Owen \\ Institute for Astronomy, University of Hawaii, 2680 Woodlawn Drive, \\ Honolulu, Hawaii 96822, USA
}

Paul Mahaffy and H. B. Niemann

Goddard Space Flight Center, Greenbelt, Maryland 20771, USA

S. K. Atreya and T. M. Donahue

Atmospheric, Oceanic and Space Sciences, University of Michigan, Ann Arbor, Michigan 48109, USA
A. Bar-Nun
Dept of Geophysics and Planetary Sciences, Tel-Aviv University, Ramat-Aviv, Israel
I. de Pater
Department of Astronomy, U. of California, Berkeley, California 94720-3411, USA

\begin{abstract}
The discovery by the Galileo Probe Mass Spectrometer that argon is enriched to the same extent as carbon and sulfur on Jupiter requires a revision of models for the formation of this giant planet. Evidently the excess heavy elements were carried to Jupiter in icy planetesimals that formed at temperatures $\leq 30 \mathrm{~K}$. This result indicates that there is no original significance in the present position of Jupiter's orbit.
\end{abstract}

\section{Introduction}

Since the recent review of this topic by Lunine et al. (2000), we have obtained a new set of results on the composition of Jupiter's atmosphere that leads to changes in some basic ideas about the chemistry of the outer solar system (Owen et al. 1999). These results were presented in a preliminary form at the Symposium and are reviewed and amplified here.

The principal change is a requirement for low temperature $(T \leq 30 \mathrm{~K})$ icy planetesimals to carry heavy elements to Jupiter. While this requirement forces a re-evaluation of the process(es) responsible for the formation of Jupiter, it seems likely that this same re-evaluation must also apply to the formation of giant and super-giant planets that are being discovered in other stellar systems. Thus it may be useful to explain how this change in perspective has occurred and to examine its consequences. 


\section{Relevance of Elemental Composition}

It has been well-established that carbon is enriched in the atmospheres of the giant planets in our solar system, by a factor that increases with the ratio of core mass (or heavy element mass) to total planetary mass (e.g. Gautier \& Owen 1989; Gautier et al. 1995). This enrichment has been understood as a consequence of the delivery of heavy elements to the forming giant planets by small, solid planetesimals (e.g. Pollack \& Bodenheimer 1989; Boss 1995; Pollack et al. 1996). Comets are often cited as the best contemporary example of what such planetesimals might have been like.

Comets are commonly regarded as extremely primitive objects that may have retained chemical abundances that existed in the interstellar cloud from which the solar system formed. Yet even these primitive objects are deficient in nitrogen compared with solar abundances (Geiss 1988; Wyckoff et al. 1991; Krankowsky 1991). The observed deficiency of nitrogen in comets has since been explained by Owen \& Bar-Nun (1995) to result from the inability of water ice - the dominant constituent of comets - to trap $\mathrm{N}_{2}$ unless the ice forms at temperatures of $30 \mathrm{~K}$ or less, whereas the comets that dominate the Oort cloud should have formed at temperatures of $55 \pm 15 \mathrm{~K}$. As $\mathrm{N}_{2}$ is the major carrier of the nitrogen in the ISM (van Dishoeck et al. 1993), it is also expected to serve the same role in the outer solar nebula. Hence if their ices cannot capture $\mathrm{N}_{2}$ efficiently, comets will be deficient in nitrogen.

This is clearly a model-dependent explanation. The model assumes the sublimation and recondensation of interstellar icy grains as they fall out of the collapsing interstellar cloud toward the mid-plane of the forming solar nebula (e.g. Lunine et al. 1991; Simonelli et al. 1997). Recondensation on the refractory cores of these grains occurs at the local ambient temperature, which is assumed to range from (approximately) $150 \mathrm{~K}$ at Jupiter's distance to (approximately) 55 $\mathrm{K}$ midway between the orbits of Uranus and Neptune. It is the Uranus-Neptune region of the solar nebula that is generally considered to be the major source of the Oort cloud comets that we enjoy in the inner solar system today (Oort 1950, 1990; Weissman 1991). Even $\sim 55 \mathrm{~K}$ is well above the $\leq 30 \mathrm{~K}$ temperature at which condensing ice is found to trap $\mathrm{N}_{2}$ completely in laboratory experiments (Bar-Nun et al. 1988).

So despite the immense distances $(\sim 50,000 \mathrm{AU})$ these comets traverse to reach us as they come in from the Oort cloud we are forced to conclude that they are not as primitive as one might think: they are depleted in the most volatile constituents that were originally present in the ISM. Besides $\mathrm{N}_{2}$, these would include argon, methane, and carbon monoxide (Owen \& Bar-Nun 1995; Notesco \& Bar-Nun 1996).

This leads to some serious consequences, because the relative proportions of nitrogen and carbon in highly volatile species in the ISM are dramatically different: whereas $\geq 70 \%$ of the nitrogen is in the form of $\mathrm{N}_{2}$-and thus not easily trapped in ice-just the reverse is true of carbon; $\geq 70 \%$ of the carbon in the ISM is in the form of relatively non-volatile species-e.g., carbon grains and organic compounds (Irvine \& Knacke 1989; Encrenaz et al. 1991; van Dishoeck et al. 1993) that are easily trapped. Thus icy planetesimals are expected to exhibit $\mathrm{C} / \mathrm{N}>$ solar. This is apparently true for comets, and was therefore expected to be true for Jupiter and other giant planets as well. Pollack \& Bodenheimer 
(1989) had also predicted a deficiency of $\mathrm{N}$ in giant planets on the basis of the subsolar values of $\mathrm{N} / \mathrm{C}$ found in comets and meteorites. The same series of laboratory experiments that demonstrated the difficulty of trapping $\mathrm{N}_{2}$ in ice forming at $T>30 \mathrm{~K}$ showed similar behavior for argon (Bar-Nun et al. 1988), suggesting that this gas should also be deficient in comets-and hence giant planets (Owen \& Bar-Nun 1995).

Considering the specific case of Jupiter, we note that conventional models for the solar nebula associate the formation of this giant planet with the distance from the sun in the solar nebula where water vapor was first able to condense, the so-called "snow-line" (e.g. van Dishoeck et al. 1993). The domain of the giant planets has been causally linked with the region of the solar nebula where ice could form (e.g. Stevenson \& Lunine 1988). Hence the surprise that greeted the discoveries of giant planets even closer to their stars than the planet Mercury is to the sun (Mayor et al. 2000).

\section{Galileo Measurements}

It was in this context that the analysis of the composition of Jupiter's atmosphere proceeded, based on measurements by the mass spectrometer carried by the Galileo Probe (Niemann et al. 1992). The initial results appeared to support the conventional model for giant planet formation. In particular, the enrichment of carbon was confirmed as $\mathrm{C} / \mathrm{H}=2.9 \pm 0.5 \times$ solar, while the discovery of $\mathrm{H}_{2} \mathrm{~S}$ led to a value of $\mathrm{S} / \mathrm{H}=2.5 \pm 0.15 \times$ solar (Niemann et al. 1998). This means that $\mathrm{S} / \mathrm{C}$ must be very close to the solar value, demonstrating that $i c y$ planetesimals had to be the carriers of heavy elements to Jupiter, as even carbonaceous chondrites have $10 \times$ less carbon relative to sulfur than the solar value (Owen 1997; Niemann et al. 1998). The early evaluation of the argon abundance gave $\mathrm{Ar} / \mathrm{H}$ $=1.7 \pm 0.6 \times$ solar $($ Niemann et al. 1996, 1998). While higher than expected, the uncertainty in this number was sufficiently large to accommodate the anticipated near-solar ratio. It was not possible to obtain a measurement of the nitrogen abundance in this first analysis of the mass spectrometer data owing to the excess $\mathrm{NH}_{3}$ delivered by the instrument's noble gas enrichment cell (Niemann et al. 1998). However, the agreement between ground-based and probe measurements of the methane abundance on Jupiter suggested that ground-based measurements could be used for ammonia as well. Those measurements indicated a value of $\mathrm{N} / \mathrm{H} \sim 1.3 \times$ solar (reviewed by Atreya et al. 1999)

A slight enrichment of $\mathrm{N}$ over the solar value was expected because of the ability of ice to trap $\mathrm{NH}_{3}$, despite its disaffinity for $\mathrm{N}_{2}$ (Owen \& Bar-Nun 1995). Thus the cometary delivery of heavy elements to Jupiter appeared to be confirmed by the Galileo Probe results.

However, an independent determination of the nitrogen abundance based on the attenuation of the probe signal by Jovian ammonia led to a discordant result of $\mathrm{N} / \mathrm{H}=3.6 \times$ solar at depths in the atmosphere with $P>8$ bars (Folkner et al. 1998). Confidence in the cometary model led to attempts to explain this discrepancy in terms of an additional microwave absorber such as $\mathrm{PH}_{3}$, but the amount required appeared prohibitive (Hoffman et al. 1999, Atreya et al. 1999). Alternatively, it was possible to show that the ground-based microwave observations of Jupiter were compatible with the Folkner et al. (1998) nitrogen 


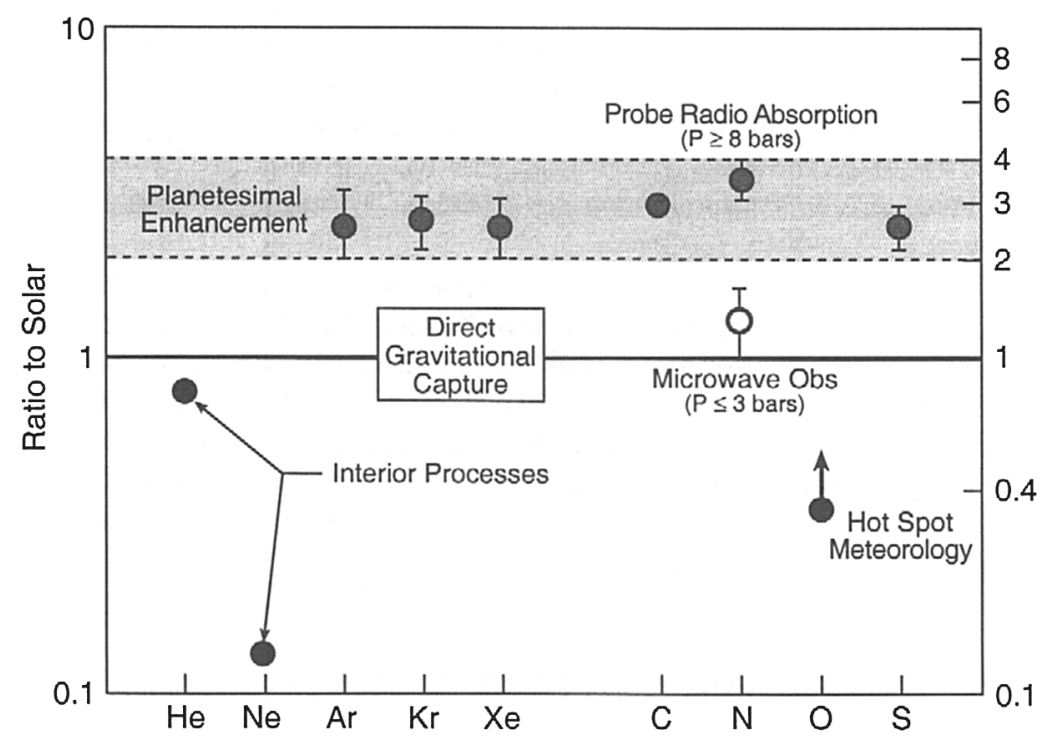

Ratio of Jovian to Solar Abundances

Figure 1. The presently available data for elemental abundances on Jupiter are plotted on a scale in which 1.0 represents solar abundances relative to hydrogen. $\mathrm{He}$ and $\mathrm{Ne}$ are depleted as they come out of solution in Jupiter's interior. Only a lower limit could be set on oxygen because the probe did not reach deep enough levels to measure $\mathrm{H}_{2} \mathrm{O}$ where it is fully mixed with the other constituents. All the other elements that were assessed show an enrichment of $3 \pm 1$ times their solar values.

abundance if ammonia in the upper troposphere of Jupiter, above the 4 bar level, was strongly depleted (Atreya et al. 1999).

Meanwhile, continued laboratory calibration of the Galileo Probe Mass Spectrometer has led to much improved determinations of noble gas abundances. The final results yield enrichments relative to solar values as follows (Owen et al. 1999; Mahaffy et al. 2000).

$$
\begin{aligned}
& { }^{36} \mathrm{Ar} / \mathrm{H}=2.5 \pm 0.5 \times \text { solar } \\
& { }^{84} \mathrm{Kr} / \mathrm{H}=2.7 \pm 0.5 \times \text { solar } \\
& { }^{132} \mathrm{Xe} / \mathrm{H}=2.6 \pm 0.5 \times \text { solar }
\end{aligned}
$$

In other words, all three of these heavy noble gases exhibit essentially the same enrichment as that found for carbon and sulfur. This is illustrated in Figure 1 from Owen et al. (1999), which includes the nitrogen value derived by Folkner et al. (1998).

We know of no solid material in the solar system that exhibits solar relative abundances of $\mathrm{C}, \mathrm{S}, \mathrm{N}$, and Ar, as illustrated in Figure 1, although we have postulated that the Kuiper Belt Objects might have such a composition, 
based on their expected formation temperatures of $T \leq 30 \mathrm{~K}$ (Owen \& Bar-Nun 1995). It is this requirement for the formation of icy planetesimals at such low temperatures that forces the abandonment of the classical "cometary" model for enriching Jupiter's heavy elements.

It also eliminates clathrate hydrates as the potential carriers of the noble gases. Lunine \& Stevenson (1985) deduced that Xe/Ar should be $>9 \times$ solar in clathrates with $\mathrm{C} / \mathrm{H} \approx 3 \times$ solar. In fact $\mathrm{Ar} / \mathrm{Xe}=$ solar, so clathrates were not involved. Instead it seems that trapping of these gases in microscopic cracks and crannies of amorphous ice forming at low temperatures as explored by Bar-Nun et al. (1988) was the dominant mechanism for getting the gases into the ice.

\section{Implications for Jupiter's Formation}

To accommodate these new results, it is necessary to think of some new scenarios for Jupiter's formation. We have proposed three possibilities (Owen et al. 1999): (a) Jupiter formed beyond $30 \mathrm{AU}$ and migrated inward to its present position. (b) The solar nebula was much colder than originally thought, so at Jupiter's present distance from the Sun the temperature was $\leq 30 \mathrm{~K}$. (c) The solid planetesimals that contributed the heavy elements to Jupiter began forming in the interstellar cloud that collapsed to make the solar nebula, and grew large enough to allow them to survive entry into the nebula.

It is interesting to note that all three of these scenarios admit the possibility of planetary systems with giant or super-giant planets at essentially any orbital distance from their respective stars. In particular, the importance of the socalled "snow-line," the radial distance in a circumstellar disk at which watervapor can first form ice, is greatly diminished. The temperature at which this condensation occurs is typically modelled at $\sim 150 \mathrm{~K}$. At this temperature, argon would be depleted by a factor $10^{6}$ compared to the amount we found on Jupiter (Bar-Nun et al. 1988).

A fourth scenario that could be envisaged involves the selective depletion of light gases in the solar nebula-decreasing the denominator instead of increasing the numerator in the element abundance ratios we have measured. A possible process for achieving this is through intense Lyman- $\alpha$ emission from the early Sun, prior to Jupiter's acquisition of its complement of light gases (N. Schwadron \& F. Adams, private communication). However, it is unlikely that the necessary amount of hydrogen could be removed without affecting the ratios of $\mathrm{He} / \mathrm{H}, \mathrm{D} / \mathrm{H}$, and ${ }^{3} \mathrm{He} /{ }^{4} \mathrm{He}$. These ratios have all been measured on Jupiter and found to be consistent with primordial values and models for the planet's interior (Mahaffy et al. 1998; Niemann et al. 1998; von Zahn et al. 1998).

Each of the three scenarios we originally proposed has its own difficulties, and we clearly need additional data to distinguish which (if any) is correct. At this stage we find the migration scenario the least likely of the three, given its requirement for large amounts of mass in the early solar nebula beyond $30 \mathrm{AU}$ and its unknown effects on the rest of the solar system. One important piece of missing information is the composition of the other three giant planets: Is Jupiter the only one that shows this enrichment in highly volatile elements, or do the others also have it? The fact that we find HCN on Neptune suggests that nitrogen is also enriched there, as it seems necessary to have $\mathrm{N}_{2}$ with an 
approximate solar abundance of $\mathrm{C} / \mathrm{N}$ in the planet's stratosphere to make the $\mathrm{HCN}$ (Marten et al. 1993). A solar value of $\mathrm{C} / \mathrm{N}$ may also be necessary to derive a reasonable (i.e., protosolar) value of $\mathrm{He} / \mathrm{H}$ from the mean molecular weight of the atmosphere (Conrath et al. 1993). However, these are both indirect arguments and the former especially begs the question, why is there no detectable HCN on Uranus? There is simply no substitute for a family of atmospheric probes that would give us the information we seek, at each planet.

A direct prediction of the Jupiter probe result is that oxygen will be as enriched as the other elements. Unfortunately, the probe did not go deep enough into the atmosphere to make this measurement, which must be done in $\mathrm{H}_{2} \mathrm{O}$. Another probe sent to a different region on the planet, where condensible species are not depleted in the upper troposphere as they were at the Galileo entry site, would settle this. In other words, the family of atmosphere probes should include Jupiter in the target list. This measurement takes on special importance in view of models that predict an outward migration of $\mathrm{H}_{2} \mathrm{O}$ in the early solar nebula by diffusion, followed by inward migration of $\mathrm{H}_{2} \mathrm{O}$-rich planetesimals subsequently formed from this water in the Jupiter region (Stevenson \& Lunine 1988; Lunine et al. 2000). This process should lead to a selective enrichment of $\mathrm{H}_{2} \mathrm{O}$ on Jupiter, which could be measured.

Another prediction is the presence of low-temperature planetesimals among the comets, particularly if all the giant planets were visited by these objects. The planet-forming process could not have been so efficient that all of these early, primitive objects were captured; some must have been scattered into the Oort cloud. If so, we will be able to identify them by their solar abundances of $\mathrm{C} / \mathrm{N}$ and $\mathrm{C} / \mathrm{Ar}$.

An interesting sidelight of this prediction is the possibility that the forming inner planets were also bombarded by these low temperature objects. The one planet that may still manifest a record of this bombardment is Venus, where the relative abundances of $\mathrm{Ar}, \mathrm{Kr}$, and $\mathrm{Xe}$ are more nearly solar than terrestrial or Martian (Donahue \& Pollack 1983). Future studies of the atmosphere of Venus will tell us just how "solar" those noble gases are, by measuring relative abundances and isotope ratios with far higher precision than previous missions could accomplish.

Acknowledgments. We thank NASA for supporting this research.

\section{References}

Atreya, S.K., Wong, M.H., Owen, T., Mahaffy, P., Niemann, H., de Pater, I., Drosssart, P., \& Encrenaz, Th. 1999, Planet Space Sci., 47, 1243

Bar-Nun, A. Kleinfeld, I., \& Kochavi, E. 1988, Phys. Rev. B, 38, 7749

Boss, A.P. 1995, Science, 267, 360

Conrath, B.J., Gautier, D., Owen, T., \& Samuelson, R.E. 1993, Icarus, 101, 168

Donahue, T.M. \& Pollack, J.B. 1983, in Venus, eds. D. M. Hunten et al. (Tucson:

Univ. Arizona Press), 1003

Encrenaz, Th., Puget, J.L., \& D'Hendecourt, L. 1991, Space Sci. Rev., 56, 83

Folkner, W.M., Woo, R., \& Nandi, S. 1998, J. Geophys. Res., 103, 22847

Gautier, D., Conrath, B.J., de Pater, I., \& Atreya, S.K. 1995, in Neptune and Triton, ed. D.P. Cruikshank (Tucson: Univ. Arizona Press), 547 
Gautier, D. \& Owen, T. 1989, in Origin and Evolution of Planetary and Satellite Atmospheres, eds. S.K. Atreya, J.B. Pollack, \& M.S. Matthews (Tucson: Univ. Arizona Press), 487

Geiss, J. 1988, Rev. Mod. Astron., 1, 1

Hoffman, J.P., Steffes, P.G., \& DeBoer, D.R. 1999, Icarus, 140, 255

Irvine, W.M. \& Knacke, R.F. 1989, in Origin and Evolution of Planetary and Satellite Atmospheres, eds. S.K. Atreya, J.B. Pollack \& M.S. Matthews (Tucson: Univ. Arizona Press), 3

Krankowsky, D. 1991, in Comets in the Post Halley Era, eds. R.L. Newburn, Jr., M. Neugebauer, \& J. Rahe (Dordrecht: Kluwer), 855

Lunine, J.I., Engel, S., Rizk, B., \& Horanyi, M. 1991, Icarus, 94, 333

Lunine, J.I., Owen, T., \& Brown, R.H. 2000, in Protostars and Planets IV, eds. V. Mannings, A. Boss, \& S. Russell (Tucson: Univ. Arizona Press), in press

Lunine, J.I. \& Stevenson, D.J. 1985, ApJS, 58, 493

Mahaffy, P.R., Donahue, T.M., Atreya, S.K., Owen, T., \& Niemann, H.B. 1998, in Primordial Nuclei and Their Galactic Evolution, eds. N. Prantzos, M. Tosi, \& R. von Steiger (Dordrecht: Kluwer), 239

Mahaffy, P.R., Niemann, H.B., Alpert, A., Atreya, S.K., Demick, J., Donahue, T.M., Harpold, D.N., \& Owen, T.C. 2000, J. Geophys. Res., in press

Marten, A., Gautier, D., Owen, T., Sanders, D.B., Matthews, H.E., Atreya, S.K., Tilanus, R.P.J., \& Deane, J.R. 1993, ApJ, 406, 285

Mayor, M., Marcy, G., Butler, \& Queloz, D. 2000, in Protostars and Planets IV, eds. V. Mannings, A.P. Boss, \& S.S. Russell (Tucson: Univ. Arizona Press), in press

Niemann, H.B., et al. 1998, J. Geophys. Res., 103, 22831

Niemann, H.B., et al. 1996, Science, 272, 846

Niemann, H.B., Harpold, D.N, Atreya, S.K., Carignan, G.R., Hunten, D.M., \& Owen, T. 1992, Space Sci. Rev., 60, 111

Notesco, G. \& Bar-Nun, A. 1996, Icarus, 122, 118

Oort, J.H. 1950, Bull. Astron. Soc. Netherlands, 11, 91

Oort, J.H. 1990, in Physics and Chemistry of Comets, eds. W.H. Huebner (Berlin: Springer), 235

Owen, T. 1997, Adv. Spa. Res., 19, 1287

Owen, T. \& Bar-Nun, A. 1995, Icarus, 116, 215

Owen, T., Mahaffy, P., Niemann, H.B., Atreya, S.K., Donahue, T., Bar-Nun, A., \& de Pater, I. 1999, Nature, 402, 269

Pollack, J.B. \& Bodenheimer, P. 1989, in Origin and Evolution of Planetary and Satellite Atmospheres, eds. S.K. Atreya, J.B. Pollack, \& M.S. Matthews (Tucson: Univ. Arizona Press), 564

Pollack, J.B., Hubickyj, O., Bodenheimer, P., Lissauer, J.J., Podalak, M., \& Greenzweig, Y. 1996, Icarus, 124, 62

Simonelli, D.P., Pollack, J.B., \& McKay, C.P. 1997, Icarus, 125, 261

Stevenson, D.J. \& Lunine, J.I. 1988, Icarus, 75, 146

van Dishoeck, E.F., Blake, G.A., Draine, B.T., \& Lunine, J.I. 1993, in Protostars and Planets III, eds. E.H. Levy \& J.I. Lunune (Tucson:U. Arizona Press), 163 von Zahn, U., Hunten, D.M., \& Lehmacher, G. 1998, J. Geophys. Res., 103, 22815

Weissman, P.R. 1991, in Comets of the Post-Halley Era, eds. R.L. Newburn, Jr., M. Neugebauer, \& J. Rahe (Dordrecht: Kluwer), 463

Wyckoff, S., Tegler, S.C., \& Engel, S. 1991, ApJ, 367, 641 


\section{Discussion}

W. $M$. Irvine: Is there any data on ${ }^{15} \mathrm{~N} /{ }^{14} \mathrm{~N}$ on Jupiter, since this might reflect the primordial solar nebular value?

T. C. Owen: There is, but it is difficult to understand. The value of ${ }^{15} \mathrm{~N} /{ }^{14} \mathrm{~N}$ is much lower than in the Sun or even on Earth. We would expect ${ }^{15} \mathrm{~N} /{ }^{14} \mathrm{~N}$ in Jupiter to be identical with the value on the Sun. It appears that there must be an error in the Jupiter determination, but no one has found it yet! Perhaps new observations by the IR spectrometer on the Cassini spacecraft next year will help solve this puzzle.

W.A. Schutte: It is likely that you can trap a large quantity of $\mathrm{N}_{2}$ (up to $\sim 10 \%$ ) in $\mathrm{H}_{2} \mathrm{O}$ ice up to temperatures well above $35 \mathrm{~K}$, if you firmly embed the $\mathrm{N}_{2}$ initially in the ice by condensation at low temperatures $(\sim 10 \mathrm{~K})$. Such a scenario would be expected if the ices were formed by condensation on grains in dense clouds.

T. C. Owen: This is exactly the point I was trying to make: in order to bring nitrogen as $\mathrm{N}_{2}$ to Jupiter in an undepleted abundance, the grains that originally trapped this $\mathrm{N}_{2}$ cannot evaporate and recondense as they pass from the ISM to the solar nebula, as traditional models require. However, these traditional models work well for the formation of comets, which are observed to be depleted in nitrogen.

M. A'Hearn: You described the difficulty in explaining terrestrial $\mathrm{D} / \mathrm{H}$ from cometary water but you also noted that we have measured $\mathrm{D} / \mathrm{H}$ only in Oortcloud comets. Could you comment on the viability of Delsemme's alternative explanation that Earth was bombarded primarily by comets formed in the Jovian region?

T. C. Owen: Comets that formed in the Jovian region might have had a $\mathrm{D} / \mathrm{H}$ ratio lower than the value in the Oort cloud if the exchange of $\mathrm{D}$ between $\mathrm{H}_{2} \mathrm{O}$ vapor and $\mathrm{H}_{2}$ could proceed rapidly enough at the ambient temperature. This should be modelled. However, such comets will also bring in a solar or nearly solar ratio of O/C. But on Earth, O/C > Solar, indicating a source of water that did not contain carbon, or much less carbon than Oort cloud comets.

H. U. Keller: Can you discuss why you think that water ice is formed on Titan?

T. C. Owen: Spectroscopy through the near-IR windows in Titan's atmosphere produces a crude spectrum that nevertheless provides useful constraints on Titan's surface composition. In particular, the fact that the albedo at $5 \mu \mathrm{m}$ is as low as that at $3 \mu \mathrm{m}$ is matched well by water ice but not by processed organic material of the type we expect to be raining out of the atmosphere. 